\title{
Philosophiques
}

Jocelyn Benoist, Robert Brisart, Jacques English, Liminaires phénoménologiques : recherches sur le développement de la théorie de la signification de Husserl , Bruxelles, Publications des Facultés universitaires Saint-Louis, 1998, 281 p.

\section{Guillaume Fréchette}

Volume 27, numéro 1, printemps 2000

Le matérialisme contemporain

URI : https://id.erudit.org/iderudit/004922ar

DOI : https://doi.org/10.7202/004922ar

Aller au sommaire du numéro

Éditeur(s)

Société de philosophie du Québec

ISSN

0316-2923 (imprimé)

1492-1391 (numérique)

Découvrir la revue

Citer ce compte rendu

Fréchette, G. (2000). Compte rendu de [Jocelyn Benoist, Robert Brisart, Jacques English, Liminaires phénoménologiques : recherches sur le développement de la théorie de la signification de Husserl, Bruxelles, Publications des Facultés universitaires Saint-Louis, 1998, 281 p.] Philosophiques, 27(1), 219-225. https://doi.org/10.7202/004922ar d'utilisation que vous pouvez consulter en ligne. 
du monde à vocation universelle, mais bien sur un regard et une perception particulière indissociable du mouvement de la passion.

Le troisième livre s'intéresse à l'autre versant du langage, à la substance phonique, c'est-à-dire à ce que Lamy, dans la métaphore qu'il emprunte à D escartes, appelle «le corps du discours ». Cette corporéité doit être analysée dans toute son étendue et avec le secours de l'ensemble des disciplines susceptible de donner à comprendre cette corporéité. Celivre va donc inclure, à côté des notions de phonétique générale, un vaste ensemble de questions relatives aux sciences, à la philosophie et à la poétique qui conduira l'auteur, dans le dernier livre, à aborder la question du style. Comme le discours est le « caractère de l'âme », il n'est pas étonnant qu'il y ait autant de différents styles qu'il y a de personnes qui parlent ou écrivent. D emême, les hommes qui vivent dans des zones climatiques différentes ont un style différent. Ici, la théorie des climats vise à rendre compte dela « couleur particulière » propreau rendu discursif dela perception. Au surplus, si Lamy reprend la tripartition reçue des styles (style sublime, style moyen et style simple), ce ne sera pas sans y ménager un espace à la passion :

lorsque les choses sont grandes, et que l'on ne peut les envisager sans ressentir quelque grand mouvement, le style qui les décrit doit être nécessairement animé, plein de mouvement, enrichi de Figures, de toutes sortes de M étaphores.

Si le sujet quel'on traite n'a rien d'extraordinaire, si on le peut considérer sans être touché de passion ; le style doit être simple (p. 378).

N égligée par les études sur la rhétorique qui ont longtemps considéré que la rhétorique française naissait avec Dumarsais et Fontanier, la R hétorique de L amy est tenue aujourd'hui, à juste titre, pour un texte essentiel Iorsqu'on s'intéresse à I'histoire des idées en général et à l'histoire de la rhétorique en particulier. Essentiel, car I'attention que Lamy porte à la question des passions dans le discours force à reconsidérer l'idée, trop souvent répandue depuis $M$ ichel Foucault, que le XVII ${ }^{\mathrm{e}}$ siècle tout entier est habité par le seul désir d'une langue qui viendrait refléter l'univocité des signes. Essentiel, parce que Lamy participe à un ample mouvement qui ira s'intensifiant au cours du siècle suivant et qui cherchera, par-delà l'enseignement de la rhétorique, à placer les phénomènes sensoriels et psychologiques au centre de la réflexion sur le langage et de la problématique esthétique. L'excellente édition de Benoît Timmermans fait plus que remettre au jour ce texte capital : elle présente surtout I'avantage de retracer les strates successives du travail de Lamy et, par là, permet de mieux comprendre le passage du «Grand Siècle » au « Siècle des Lumières ».

LUCIE DESJARDINS

Département d'études littéraires Université du Québec à Montréal

Jocelyn Benoist, Robert Brisart, Jacques English, Liminaires phénoménologiques : recherches sur le développement de la théorie de la signification de Husserl. Bruxelles, Publications des Facultés universitaires Saint-Louis, 1998, 281 p.

Cet ouvrage, dont le thème demeure encore inusité dans la littérature de langue française, tente d'investiguer à nouveaux frais la théorie de la signification de H usserl. En 
effet, sur la base des publications des dernières années de la collection des $\mathrm{H}$ usserliana, plusieurs efforts de relecture de la première partie de l'œuvre deH usserl ont été entrepris, auxquels il faut dire que les auteurs du présent ouvrage ont contribué dans une mesure importante chez les francophones. C'est donc essentiellement sur cette première partie de l'œuvre de H usserl qu'insistent les auteurs, celle que couvre la rédaction de la thèse d'habilitation de 1887 (Über den Begriff der $Z$ ahl) jusqu'aux premières ébauches du vocabulaire noématique en 1911/1912. Cette période voit paraître des ouvrages comme la Philosophie de l'arithmétique (1891), les R echerches logiques (1900/1901), ainsi que des leçons telle que la Théorie de la signification (1908), qui sont de première importance quant au thème de la signification, en ce qu'ils tentent à la fois de définir le statut de la signification à l'intérieur même de la relation intentionnelle, mais également de faire composer une analyse linguistique et une grammaire dans l'élaboration du langage d'une ontologie.

Dans « $\mathrm{L}$ a découverte des processus signitifs dans la première œuvre de H usserl (1887-1891) » (p. 9-62), Brisart insiste sur le passage des processus abstractifs, issus del'abstraction mathématique, aux processus signitifs, un passage qui manifesterait la première élaboration d'une théorie de la signification chez H usserl : « c'est par la découverte des processus signitifs intrinsèques à l'arithmétique et, avec elle, par la mise en évidence de la distinction cardinale entre signification et intuition, que s'est décidée pour la première fois la mise en perspective phénoménologique de l'objet intentionnel en tant qu'objet idéal » (p. 10). En d'autres termes, cette découverte des processus signitifs par H usserl est à considérer comme inaugurant celle qui l'amène à distinguer le contenu de la représentation : le passage à une conception symbolique de la représentation du nombre dans la Philosophie de l'arithmétique est motivé par l'insuffisance d'une définition del'acte de dénombrer en tant que processus abstractif, insuffisance qui permet seulement une synthèse des concepts de nombre au niveau des abstracta. A ussi, une représentation du nombre de ce point de vue se composerait de deux parties psychologiques, soit le processus d'unification collective (synthèse donnant les abstracta) et le quel que chose. Cette distinction laisse toutefois indéterminée la question du contenu des représentations : dit autrement, les nombres sont représentés selon cette première conception comme du quelque chose, « au sens où quelque chose est une formation propre à un acte psychique qui se représente un contenu quel qu'il soit, abstraction faite de la teneur particulière de ce contenu » (p. 19). La représentation du nombre serait donc une représentation sans contenu ou, dit autrement, un acte qui se représente l'ensemble (qui lie de manière collective), ce qui expliquerait l'acte de dénombrer comme indépendant de ce qui se trouve dans le contenu de la représentation. Comme on le sait, cette question de la représentation est posée par H usserl sur un terrain prioritairement mathématique, cette thèse d'habilitation de 1887 visant en quelque sorte à expliciter l'intuition de Weierstrass selon laquelle un développement rigoureux de l'analyse supérieure doit provenir de l'arithmétique élémentaire. C'est là un point que l'auteur aurait pu gagner à expliciter davantage, car il est souvent de mise de considérer les premiers écrits de $\mathrm{H}$ usserl à la lumière de son intérêt pour la philosophie brentanienne, alors qu'on oublie parfois que cet intérêt était motivé par les questions de fondement des mathématiques qu'il dével oppait sous I'influence de Weierstrass. Q uant à la conception symbolique de la représentation du nombre, elle fait passer l'analyse des nombres du point de vue psychologique à un point de vue dit signitif ou symbolique, ce que l'auteur explique par le fait queles opérations numériques en arithmétique n'opèrent sur les nombres qu'en tant que signes 
ou symboles de concepts, et que ce sont seulement ces signes qui sont liés dans le calcul. Bien loin de selaisser dériver des concepts de l'arithmétique élémentaire, les nombres irrationnels apparaissent plutôt comme relevant de concepts symboliques dont une justification et une fondation ultimes n'auraient donc pu simplement en appeler à une analyse du concept de nombre au sens le plus élémentaire. En ce sens, I'approche seulement symbolique doit être d'un genre différent del'approche psychologiquedescriptive. Par l'élaboration de cette distinction, l'auteur souhaite insister sur le fait que c'est par une réflexion sur les processus signitifs et leur rôle dans la construction de la représentation que $\mathrm{H}$ usserl se détache pour la première fois et de manière significative d'une tendance psychologiste à la Brentano. Aussi, la Philosophie de l'arithmétique ne serait pas si brentanienne si nous acceptons avec l'auteur qu'à partir de 1890, H usserl se détache progressivement d'une conception pratique de la logique, comme Kunstlehre, et qu'en ce sens, il n'aurait jamais pu être pleinement psychologiste car c'est d'abord eu égard à la question de la discipline fondatrice (arithmétique ou logique) que la psychologie aurait été avancée. $M$ ais dans la mesure où la conclusion de la Philoso phie de l'arithmétique suggère une dépendance de l'arithmétique à la logique, la question du psychologisme dela Philosophie de l'arithmétique n'est pas centrale, mais c'est plutôt celle de la critique de l'al gébrisation de la logique (telle que proposée par Schröder à l'époque) qui serait déterminante. Or, selon l'auteur, la critique de l'algébrisation schröderienne de la logique, telle qu'elle fera l'objet d'un compterendu de H usserl en 1890 et sur lequel l'auteur nous promet un exposé paru récemment dans la revue $R$ echerches husserliennes, serait déjà une critique de la logique comprise comme Kunstlehre. Partant de ce fait, il est difficile de concevoir quelle thèse est véritablement défendue dans la Philosophie de l'arithmétique : si l'ouvrage donne un rôle prioritaire à la logique sur l'arithmétique, c'est dans la mesure où cette logique est une science pratique dont les tâches sont prescriptives et non normatives. Si cette conception contrevient à l'algébrisation de la logique, cette contravention ne signifie pas pour autant que H usserl accorde à la logique un rôle de science théorique aux lois aprioriques. D ans les faits, la Philosophie del'arithmétique marque peut-être la fin de l'étude psychologique de l'origine des nombres, non pas parce que les moyens d'investigation ne sont pas efficaces, mais parce que l'objet d'étude n'est plus considéré de façon aussi fondamentale qu'il l'était dans les années précédentes. De cette façon, il serait difficile de considérer le passage à une étude psychologique de la logique comme foncièrement moins psychologique qu'une étude psychologique de I'arithmétique, ce que l'auteur doit soutenir de quelquefaçon pour supporter la thèse selon laquelle le passage à l'étude des processus signitifs par H usserl dans la Philosophie de l'arithmétique de 1891 est un abandon de la psychologie descriptive et des prémisses psychologistes grevant une période brentanienne qu'il aurait commencée à dépasser en 1891, pour ainsi affirmer que l'apparition de la problématique signitive est le premier pas vers la phénoménologie en ce que celle-ci est foncièrement anti-psychologiste, ce que l'auteur n'affirme pas précisément mais qui est visiblement la conclusion de l'argumentation qu'il propose. D'autre part, il est étonnant de voir que Brisart tente de défendre une lecture anti-frégéenne de cette portion de l'œuvre de H usserl, en faisant abstraction des travaux suscités par cette perspective : nous pensons ici aux polémiques suscitées par Føllesdal, au sujet desquelles ont notamment pris parti M ohanty, Sokolowski et W illard, en défendant unelecture du premier H usserl à propos de laquelle il aurait été intéressant de voir Brisart prendre explicitement position. Dans la suite de Føllesdal, Smith et M clntyre ont insisté quant à eux sur 
I'explication du concept de noème dans les mêmes termes que la notion frégéenne de sens (Sinn), et les différentes polémiques suscitées par les premiers travaux de Føllesdal auront contribué dans une très large mesure à réinvestir le champ des recherches husserliennes partant du thème de la signification, ce qui est tout simplement passé sous silence dans l'article de Brisart.

Dans son long article sur « Les trois étapes de la formation de la théorie de la signification chez le premier H usserl » (p. 63-218), English retracel'évolution du thème de la signification de la Philosophie de l'arithmétique aux L eçons de 1908 en passant par les Recherches logiques. Tout comme Brisart l'avait fait, English insiste également sur le fait que le manuscrit de 1890 est une première élaboration de la théorie de la signification que l'on retrouvera dans la Philosophie de l'arithmétique un an plus tard: partant de certaines prises de position psychologistes sur la nature des nombres, Husserl était encore confronté, dans la Philosophie de l'arithmétique, à l'élaboration d'une théorie de la signification sur le modèle des concepts de nombres propres, et donc une théorie de la signification fonctionnant sur une modal ité symbolique « au sens du remplacement ou de la substitution » (p. 65), modalité réductrice à celle de la perceptivité selon English, en ce que cette substitution ne permettait pas à l'intentionnalité de se dégager du type de structuration de la modalité perceptive de signification. Par cette modalité, English entend une définition de la signification en termes de désignation (Bezeichnung) à l'opposé de la signification à proprement parler (Bedeutung). Selon English, il y aurait aussi deux ontologies rattachées à cette théorie de la signification passant de la modalité perceptive à la modalité signitive, et donc de la première à la seconde étape annoncée par le titre de l'article : « Si la première théorie de la signification proposée par H usserl de 1887 à 1891, et exposée dans la Philosophie de l'arithmétique, avait donc souffert d'une grave insuffisance, c'était sans aucun doute à cause de cette absence de toute garantie ontologique qui aurait été aussitôt fournie par la réfé rence à un pôle objectif véritablement transcendant » (p. 93). A ussi, le problème de cette première théorie de la signification réside selon English dans l'incapacité de H usserl à assurer un sol ontologique transcendant aux significations ou désignations de ce qui est donné dans la modalité perceptive. $M$ ais English se fait vraisemblablement très dur, dans la mesure où la préoccupation de H usserl dans la Philosophie de l'arithmétique n'est pas tant de fournir une théorie de la signification telle qu'elle sera entendue dans l'optique réaliste des R echerches logiques, où même à la lumière des $L$ eçons de 1908, mais plutôt de définir le statut des nombres et leur signification comme entités psychologiques puis, plus tard, comme signes. M ais qu'en est-il de cette seconde étape dans le développement de la théorie de la signification husserlienne? Selon English, $\mathrm{H}$ usserl serait parti dans la première étape de sa théorie de la signification par l'affinité originaire entre les vécus intérieurs et l'ontologie matérielle pour s'élever ensuite aux vécus supérieurs et à l'ontologie formelle, puis en redescendant vers le bas tout en conservant les résultats établis par le processus abstractif (p. 95-96), en conservant donc de cette façon un parallélisme entre le matériel et le formel, mais en ne changeant pas de modalité mal gré le changement de régime. Autrement dit, la signification demeurerait sur le niveau de la modalité perceptive, bien que les ontologies atteintes auraient exigé, selon English, une modalité différente, plus « objective». Ce n'est toutefois pas ici la première étape telle qu'envisagée par English car, selon lui, c'est par des changements dans les modalités mêmes del'intentionnal ité qu'une théorie dela signification peut être amenée à se modifier. II s'agit alors du mode d'exercice de l'intentionnalité, qui pour les concepts formels doit ajuster son tir, et nommément passer à un mode signitif. 
$M$ ais cette évolution de la première étape (Philosophie de l'arithmétique) à la seconde (Recherches logiques) marque également le développement d'un motif qui, bien que English n'emploie pas le mot, pourrait être qualifié de dialectique, en ce que le jeu qui s'institue entre les facteurs subjectifs qui relèvent du signifier et les facteurs objectifs que forment les significations crée « une espèce d'aller et retour perpétuel, dont l'étude devrait donc constituer désormais, et selon un régime de conditionnement bilatéral, s'exerçant sur une zone mixte, fondée des deux côtés, le centre même de toute théorie possible de la signification » (p. 125). En ce sens, il faut comprendre que « si la théorie de la signification doit donc trouver désormais son centre de gravité, ce ne peut être que sur la zone médiane même où doivent venir interférer ces deux séries dissymétriques de variations, les unes offertes par cet énorme pouvoir de modification dont l'intentionnalité se trouve investie subjectivement, en ayant toujours les moyens de changer ses genres de rapports aux objets qu'elle peut viser audelà d'elle-même selon les différents actes par lesquels elle décide de les rejoindre, et les autres qui sont fournies, elles, par toutes les possibilités de combinaisons inhérentes aux structures essentiellement syncatégorématiques du langage avec les lois de transformation grammaticales qui leur sont propres »(p. 129). C'est ce qui expliquerait le troisième moment de la théorie husserlienne de la signification : il y aurait en 1908 un « renversement de perspective qui déplace le centre de gravité de toute cette problématique de l'ontologie vers la phénoménologie» (p. 143). Cependant, cette étape maintient en place les distinctions des deux premières étapes, mais va aussi les soumettre à une série de réélargissements caractéristiques, en procédant toutefois à une grande avancée, consistant à ne pas confondre les modes de visée signitifs et perceptifs. Enfin, ce problème de la théorie de la signification, bien qu'il semble achevé et substantiel dans ce texte de 1908, ne se distingue jamais du problème du développement de l'intentionnalité, trouble que manifeste le manuscrit de 1911 sur les significations empiriques, qui vient remettre en question la légitimité d'une systématique des actes intentionnels dans le domaine déictique.

Contrairement à Brisart, English se limite à un exposé plus descriptif qu'explicatif, bien que celui-ci vise à soutenir que le développement des thèmes de la phénoménologie transcendantale est effectif dès 1894, certes à travers la polémique entre H usserl et Twardowski sur le contenu des représentations, mais également en affirmant qu'il y avait dès cette époque un enjeu phénoménologique dans le passage d'une modalité perceptive à une modalité signitive ( $p .193$ et suivantes) pour la signification, ce qui peut être une thèse valable, mais qui mériterait pour cela d'être étudiée à la lumière d'une définition plus précise de la phénoménologie, ce que English croit d'emblée acquis chez ses lecteurs.

Enfin, le troisième et dernier article est celui de J. Benoist (« L'identité d'un sens : $\mathrm{H}$ usserl, des espèces à la grammaire », p. 219-271), qui tente de poursuivre une des idées développées dans son dernier livre (Phénoménologie, sémantique, ontologie), selon laquelle les R echerches logiques de H usserl se déploient dans un champ métaphysique neutre dépassant, d'une part, le débat idéalisme/réalisme, et ne manifestant, d'autre part, aucun engagement métaphysique. Cette idée se poursuit ici sur la base de la relation entre la grammaire et la signification, comme quoi la signification telle qu'elle est définie dans les Recherches logiques se complète par la grammaire, car comme Benoist le remarque, «le problème est celui de la parole elle-même comme contexte, de l'occasion inhérente à la parole qui se manifeste dans ce phénomènefondamental qui est celui de son indexicalité, phénomène que $H$ usserl reconnaît 
assurément comme fondamental, et qui l'embarasse fort, justement parce que lui se situe dans son analyse à ce niveau même qui est celui des actes du signifier, qui est aussi le niveau de cette occasionnalité » (p. 225).

Benoist insiste ici sur un problème auquel sera confronté H usserl de 1901 à 1908 quant à sa théorie de la signification, soit la place des significations empiriques. Si la signification est itérée dans l'acte, elle existe en un certain sens indépendamment de lui, au sens où l'acte n'est pas la signification, mais dans la mesure où la signification est obtenue dans l'acte, ce qui n'est pas sans rappeler un certain réalisme platonicien en ce que $\mathrm{H}$ usserl discute des significations comme d'entités, mais qui dans les faits n'a que très peu à faire avec le réal isme, en ce que les actes doivent être itérés, performés, et que cette clause performative a plus à faire avec un certain pragmatisme qu'avec un réalisme. Benoist ajoute : «Dans les Recherches logiques, le problème demeure de penser les formes d'unité d'une intentionnal ité - celle de la signification - qui n'est pas tournée vers sa propre unité, mais vers celle de l'objet, qui est visé - même si celle-ci bien sûr ne pourra s'attester que dans celle-là, qui y constitue la voie d'accès obligée. La réponse tient dans la théorie des espèces de signification, qui ne sont rien d'autre alors que les formes d'unité de la signification » (p. 228).

L'auteur fait ici un parallèle entrele rouge in specie et les différents moments de rouge sur des bandes de papier rouge: si nous pouvons considérer la signification comme le rouge in specie, comme $\mathrm{H}$ usserl tente à première vue de nous le faire croire, on retrouverait la même signification dans différents énoncés comme le même rouge dans différents objets. $M$ ais comme je ne vois pas le rouge comme je vois la signification, cette différence appuierait en un certain sens la distinction des genres signitifs, perceptifs et abstractifs dans la phénoménologie. $M$ ais comme Benoist le mentionne en faisant référence à $\mathrm{R}$ igal, le problème de l'identité sensible se rapproche de celui de l'identité de la signification, en ce que le caractère fondamentalement sémantique du problème de l'identité sensible s'y révèle comme n'étant pas celui de la perception de nuances du « même » rouge mais comme étant celui de la perception de nuances différentes de rouges comme mêmement rouges : « Cette généralité [de la signification], en tant qu'idéalité, n'est général ité d'aucune généralisation (communisation), mais bien plutôt d'un manque, d'une absence d'inhérence à aucun acte qui est pourtant constitutive du sens de ces actes mêmes, et, faut-il dire rien que cela - c'est ce qui fait le statut irréductiblement phénoménologique de la signification dans les Recherches logiques, que critiquera l'exposé de 1908 » (p. 235). L'auteur conclut cette première partie en insistant sur le fait que les entités idéales que sont les significations dans les R echerches logiques ne sont pas des idéalités platoniciennes. Bien qu'il s'agisse d'entités idéales, elles sont d'abord et avant tout opératoires.

En deuxième partie, l'auteur met de l'avant la nécessité manifeste dans les L eçons de 1908 de donner une certaine consistance ontologique à la signification : «C'est donc au moment où le platonisme de la signification au sens bolzanien du terme s'accuse que l'on est peut-être le plus loin du platonisme comme doctrine de l'idée. II n'y a pas de transcendance où le sens pourrait déployer son manque, mais juste le plein, à vrai dire absolument vide (au sens de vide d'intuition, indifférent à elle) des formes de signification, dans leur conjugaison possible» (p. 258). Cette « objectivation » de la signification amène alors $\mathrm{H}$ usserl à radicaliser la distinction entre objets idéaux et objets empiriques, rendant la signification empirique inséparable du monde où elle apparaît, et ne la rabattant pas sur l'absence de limites de la raison objective, comme c'était le cas dans les R echerches logiques. Sans prendre 
explicitement position sur le sens à donner ainsi que sur la valeur des différentes modifications que $\mathrm{H}$ usserl applique à sa théorie de la signification, il faut toutefois noter que l'article de Benoist prend des précautions louables quant à une explication de ces modifications « en zigzag », telle que la suggère trop allusivement English.

Enfin, il est dommage que le premier ouvrage en langue française sur la litigieuse question de la signification dans la première portion de l'œuvre de $\mathrm{H}$ usserl n'ait pas du tout tenté de comprendre le rôle joué par la psychologie descriptive dans le développement des R echerches logiques ainsi que dans son rapport à la théorie de la signification, une méthode que tire $\mathrm{H}$ usserl de l'enseignement de Brentano et qui motive sans doute plus qu'on ne le pense l'élaboration de sa phénoménologie dans la période couverte par l'ouvrage étudié ici.

GUILLAUME FRÉCHETTE

Université du Québec à Montréal 